\title{
Network Reliability Assessment in a Random Environment
}

\author{
S. Özekici, ${ }^{1}$ R. Soyer ${ }^{2}$ \\ ${ }^{1}$ Department of Industrial Engineering, Koç University, 80910 Sarlyer-İstanbul, Turkey \\ ${ }^{2}$ Department of Management Science, The George Washington University, \\ Washington, DC 20052
}

Received 7 May 2001; revised 3 September 2002; accepted 21 November 2002

DOI 10.1002/nav.10072

\begin{abstract}
In this paper we consider networks that consist of components operating under a randomly changing common environment. Our work is motivated by power system networks that are subject to fluctuating weather conditions over time that affect the performance of the network. We develop a general setup for any network that is subject to such environment and present results for network reliability assessment under two repair scenarios. We also present Bayesian analysis of network failure data and illustrate how reliability predictions can be obtained for the network. @ 2003 Wiley Periodicals, Inc. Naval Research Logistics 50: 574-591, 2003. in Wiley InterScience (www.interscience.wiley.com). DOI 10.1002/nav.10072
\end{abstract}

Keywords: network reliability; power systems; random environment; Bayesian analysis; Markov chain

\section{INTRODUCTION AND OVERVIEW}

In this paper we consider networks that consist of components operating under a randomly changing common environment. Our work is motivated by power system networks that are subject to fluctuating weather conditions over time that effect the performance of the network. The affect of environmental conditions on reliability of power networks have been recognized in earlier papers by Gaver, Montmeat, and Patton [5] and Billinton and Bollinger [2], where the authors pointed out that power systems networks are exposed to fluctuating weather conditions and that the failure rates of equipment and lines increase during severe environmental conditions. These earlier papers proposed use of a two-state Markov model to describe normal and severe weather conditions and presented reliability results for parallel and series systems with small number of components. A more recent overview of these models and their extensions can be found in Billinton and Allan [1].

Özekici [12] discusses complex stochastic models where the deterministic and stochastic model parameters change randomly with respect to a randomly changing environmental factor. Thus, these parameters can be viewed as stochastic processes rather than simple deterministic

Correspondence to: S. Özekici

(C) 2003 Wiley Periodicals, Inc. 
constants. He provides many other examples involving queueing, inventory, and reliability models. In the present setting, we consider a periodic-review reliability model where each component of the network either fails or survives in a given period. The probability of survival depends on the state of the environment in which the components operate. We consider two possible repair scenarios: Either failed components are replaced by identical ones only if the network fails (minimal repair), or any component that fails is replaced immediately by an identical one (maximal repair).

The proposed model extends available literature on reliability theory where failure probabilities of the devices change with respect to a randomly changing environment. The reader is referred to Çınlar and Özekici [4] for a detailed discussion on this idea, and further results presented, for example, by Lefèvre and Milhaud [9], Singpurwalla and Youngren [16], Singpurwalla [15] and Özekici [11]. In our approach, we use a discrete-time setting which allows us to obtain useful and practical computational formulas on complex network reliability assessment. We also present a Bayesian approach for network reliability assessment under this model using Markov Chain Monte Carlo methods and in so doing make a contribution to the literature in Bayesian analysis of network reliability. See Lynn, Singpurwalla, and Smith [10] for the state-of-the-art in this area.

The details of the network reliability model is presented in Section 2. Section 3 is devoted to its stochastic analysis under both repair scenarios. This culminates in explicit computational procedures and formulas for network reliability and the mean-time-to-failure (MTTF). In Section 4, we illustrate how these results can be used if uncertainty about model parameters are described by a prior distribution in a Bayesian setup. We present posterior analysis of network failure data and illustrate how reliability predictions can be obtained in Section 5. This analysis is presented both for the case when the environment is observable and unobservable.

\section{A MODEL FOR NETWORK RELIABILITY}

Consider a network with $K$ components with an arbitrary structure function $\phi$ and reliability function $h$. The components of the network are observed periodically at times $t=1,2, \cdots$ and the state of component $k$ at time $t$ is

$$
X_{t}(k)= \begin{cases}1, & \text { if component } k \text { is functioning at time } t \\ 0, & \text { if component } k \text { is not functioning at time } t\end{cases}
$$

The state of the reliability system, or the network, at time $t$ is represented by the vector $\mathbf{X}_{t}=$ $\left(X_{t}(1), X_{t}(2), \ldots, X_{t}(K)\right)$ and the binary variable $\phi\left(\mathbf{X}_{t}\right)$ is equal to 1 if and only if the network is functioning at time $t$. We will let $L_{k}$ denote the lifetime of component $k$ while $L$ denotes the lifetime of the system. It is well known that if the network is a serial connection of components, then

$$
\phi\left(\mathbf{X}_{t}\right)=\prod_{k=1}^{K} X_{t}(k)
$$

and 


$$
L=\min _{k=1,2, \ldots, K} L_{k}
$$

whereas, in a parallel configuration, we have

$$
\phi\left(\mathbf{X}_{t}\right)=1-\prod_{k=1}^{K}\left(1-X_{t}(k)\right)
$$

and

$$
L=\max _{k=1,2, \ldots, K} L_{k}
$$

The reliability model is modulated by an environmental process $Y=\left\{Y_{t} ; t \geq 1,2, \cdots\right\}$ which is assumed to be a Markov chain with a discrete state space $E$ and transition matrix $\mathbf{P}$. The process $Y$ is central to our model since the survival probability of any component $k$ at any time $t$ depends only on the prevailing environment such that

$$
P\left[X_{t}(k)=1 \mid Y_{t}=i\right]=\pi_{k}(i)
$$

In other words, if the environmental process $Y$ is in state $i$, then component $k$ survives the period with probability $\pi_{k}(i)$. Given the environmental process $Y$, we assume that the survival probabilities for the next period depend on $Y$ only through its current state rather than on its whole history. Moreover, the states of the components at different time epochs are conditionally independent given $Y$. This model was first analyzed by Özekici [13] in the context of a Markov modulated Bernoulli process where the success probability depends on a randomly changing environment. He provides a rather complete analysis on the transient and ergodic behavior of such processes.

It follows from (6) that the life distribution of component $k$ is characterized by

$$
P\left[L_{k}>m \mid Y\right]=\prod_{t=1}^{m} \pi_{k}\left(Y_{t}\right)
$$

since the component must survive the first $m$ time periods. Moreover, we assume that, given the environment, the component lifetimes are conditionally independent so that

$$
P\left[L_{1}>m_{1}, L_{2}>m_{2}, \ldots, L_{K}>m_{K} \mid Y\right]=\prod_{k=1}^{K} \prod_{t=1}^{m_{k}} \pi_{k}\left(Y_{t}\right) .
$$

Given the environmental process $Y$, the reliability of the network at time $t$ is given by

$$
E\left[\phi\left(\mathbf{X}_{t}\right) \mid Y\right]=h\left[\boldsymbol{\pi}\left(Y_{t}\right)\right]
$$


where $h \equiv E[\phi(X)]$ is the reliability function corresponding to $\phi$ and $\pi(i)=\left(\pi_{1}(i), \ldots\right.$, $\left.\pi_{K}(i)\right)$ is the vector of survival probabilities in environment $i$. As examples, for the series system in (2), we have

$$
h\left[\boldsymbol{\pi}\left(Y_{t}\right)\right]=\prod_{k=1}^{K} \pi_{k}\left(Y_{t}\right)
$$

while for the parallel system in (4) this becomes

$$
h\left[\boldsymbol{\pi}\left(Y_{t}\right)\right]=1-\prod_{k=1}^{K}\left(1-\pi_{k}\left(Y_{t}\right)\right) .
$$

We will denote the set of all components that are functioning prior to period $t$ by $Z_{t}$ such that $Z_{1}$ is the set of all functioning components at the outset and

$$
Z_{t+1}=\left\{k=1,2, \ldots, K ; X_{t}(k)=1\right\}
$$

is the set of components that survive period $t$ for all $t \geq 1$. The state space of the stochastic process $Z=\left\{Z_{t} ; t=1,2, \cdots\right\}$ is the set of all subsets of the component set $\mathscr{K}=\{1,2, \ldots$, $K\}$. Although it is not required in the following analysis, it is reasonable to assume that $Z_{1}=$ $\mathscr{K}$. Moreover, it follows from the stochastic structure explained above that

$$
P\left[Z_{t+1}=M \mid Z_{t}=S, Y_{t}=i\right] \equiv Q_{i}(S, M)=\prod_{k \in M} \pi_{k}(i) \prod_{k \in\left(S \cap M^{c}\right)}\left(1-\pi_{k}(i)\right)
$$

for any subsets $M, S$ of $\mathscr{K}$ with $M \subseteq S$. In words, $Q_{i}(S, M)$ is the probability that the set of functioning components after one period will be $M$ given that the environment is $i$ and the set of functioning components is $S$. This function will play a crucial role in our analysis of the network. The stochastic structure of our network reliability model is made more precise by noting that, in fact, the bivariate process $(Y, Z)$ is a Markov chain with transition matrix

$$
P\left[Z_{t+1}=M, Y_{t+1}=j \mid Z_{t}=S, Y_{t}=i\right] \equiv P(i, j) Q_{i}(S, M)
$$

for any $i, j \in E$ and subsets $M, S$ of $\mathscr{K}$ with $M \subseteq S$. In many cases, it is best to analyze network reliability and other related issues using the Markov property of the chain $(Y, Z)$.

Denote the set structure function $\Psi$ by

$$
\Psi(M)=\phi(m)
$$

where $m=\left(m_{1}, m_{2}, \ldots, m_{K}\right)$ is the binary vector with $m_{k}=1$ if and only if $k \in M$. Then,

$$
q_{i}(S)=\sum_{M \subseteq S, \Psi(M)=1} Q_{i}(S, M)
$$


is the conditional probability that the network will survive one period in environment $i$ given that the set of functioning components is $S$. The characterization in (16) can be written in terms of the path-sets of the network. Let $\mathscr{P}$ denote the set of all combinations of components that makes the network functional. In other words,

$$
\mathscr{P}=\{M \subseteq \mathscr{K} ; \Psi(M)=1\}
$$

then (16) becomes

$$
q_{i}(S)=\sum_{M \subseteq S, M \in \mathscr{P}} Q_{i}(S, M)
$$

and

$$
q(i)=q_{i}(\mathscr{K})=\sum_{M \in \mathscr{P}} Q_{i}(\mathscr{K}, M)=\sum_{M \in \mathscr{P}} \prod_{k \in M} \pi_{k}(i) \prod_{k \in M^{c}}\left(1-\pi_{k}(i)\right)
$$

is the probability that the network, with all components functioning, will survive one period in environment $i$.

Although our model supposes that the transition probability matrix $Q$ has the structure given by (13), we would like to point out that this choice is made purely for computational tractability. The results that follow are still true for any $Q$ that properly describes the probabilistic structure of failures. As an illustration of this idea, consider the network structure given in Figure 1 consisting of three components. For example, in a power distribution system, components 1 and 2 may represent two high voltage transmission lines carrying electricity to a given location where component 3 is a transformer that reduces the voltage. In a manufacturing system, components 1 and 2 may be two machines working in parallel where component 3 is another machine that completes the production. Although this network and the numerical illustration that will be presented in the last section has only a few components, our results still apply to much larger systems. The size is kept small for illustrative purposes only.

Supposing for simplicity that there are only two environmental states, the survival probabilities are given by six parameters $\left\{\pi_{k}(i) ; i=1,2\right.$ and $\left.k=1,2,3\right\}$. Take, for example, $S=$ $\mathscr{K}=\{1,2,3\}$ and $M \in \mathscr{P}=\{\{1,2,3\},\{1,3\},\{2,3\}\}$; the reliability structure prescribed by (13) gives

$$
Q_{i}(S, M)= \begin{cases}\pi_{1}(i) \pi_{2}(i) \pi_{3}(i), & \text { if } M=\{1,2,3\} \\ \pi_{1}(i) \pi_{3}(i)\left(1-\pi_{2}(i)\right), & \text { if } M=\{1,3\}, \\ \pi_{2}(i) \pi_{3}(i)\left(1-\pi_{1}(i)\right), & \text { if } M=\{2,3\}\end{cases}
$$

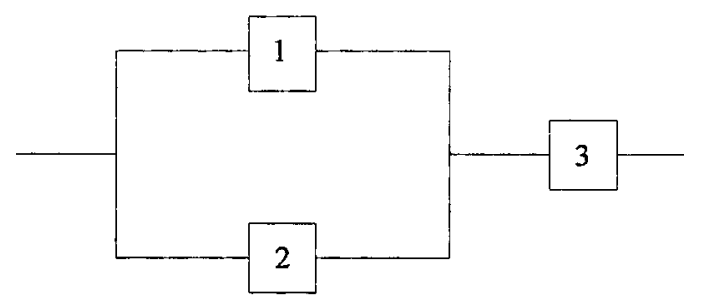

Figure 1. A simple network. 
and (19) yields

$$
\begin{aligned}
q(i) & =\pi_{1}(i) \pi_{2}(i) \pi_{3}(i)+\pi_{2}(i) \pi_{3}(i)\left(1-\pi_{1}(i)\right)+\pi_{1}(i) \pi_{3}(i)\left(1-\pi_{2}(i)\right) \\
& =\pi_{3}(i)\left[1-\left(1-\pi_{1}(i)\right)\left(1-\pi_{2}(i)\right)\right] .
\end{aligned}
$$

The transition probabilities can be obtained in a similar manner for other $S$ and $M$.

However, in many real life applications, the failure structure may be more complex than it is assumed in (20). For example, it may be such that, as soon as one of the two parallel components fail during the period, the other one may be more prone to failure. This is indeed the case in power distributions systems where parallel lines share the total load. If a line fails, then the other line will have to carry more load, and this will increase the probability of its failure. Suppose that the decreased survival probabilities are given by two additional parameters $\bar{\pi}_{1}(i) \leq \pi_{1}(i)$ and $\bar{\pi}_{2}(i) \leq \pi_{2}(i)$ given that the other parallel component has failed. In our analysis, it now suffices to take

$$
Q_{i}(S, M)= \begin{cases}\pi_{1}(i) \pi_{2}(i) \pi_{3}(i), & \text { if } M=\{1,2,3\}, \\ \bar{\pi}_{1}(i) \pi_{3}(i)\left(1-\pi_{2}(i)\right), & \text { if } M=\{1,3\}, \\ \bar{\pi}_{2}(i) \pi_{3}(i)\left(1-\pi_{1}(i)\right), & \text { if } M=\{2,3\},\end{cases}
$$

and (19) now yields

$$
\begin{aligned}
q(i) & =\pi_{1}(i) \pi_{2}(i) \pi_{3}(i)+\bar{\pi}_{1}(i) \pi_{3}(i)\left(1-\pi_{2}(i)\right)+\bar{\pi}_{2}(i) \pi_{3}(i)\left(1-\pi_{1}(i)\right) \\
& =\pi_{3}(i)\left[\pi_{1}(i) \pi_{2}(i)+\bar{\pi}_{1}(i)\left(1-\pi_{2}(i)\right)+\bar{\pi}_{2}(i)\left(1-\pi_{1}(i)\right)\right] .
\end{aligned}
$$

At this point, note that the structures of (22) are (23) are different than those of (13) and (19). This is due to the fact that now the survival probability of the parallel component 1 or 2 depends on the survival or failure of the other parallel component. Our technical analysis in the following sections requires only that $Q_{i}(S, M)$ is known parametrically and that the environment changes according to a Markov chain. There may in fact be interdependencies among the components as in (22). Our choice of (13) is a stylish one that provides computational tractability in both Markovian and Bayesian analysis.

The load-sharing effect is in fact quite crucial in power distribution networks. Our model can be used to accommodate this generalization by defining the environment accordingly. The environment is a generic term here that does not necessarily relate only to the weather conditions that affect the failure probabilities. It may as well represent the demand structure so that, in our example, if $i=1$ is the "normal load" state, then (20) may apply since the effect of load-sharing is negligible. However, if $i=2$ is the "heavy load" state, then the transition probabilities may be given by (22). The crucial assumption is that the environmental process is external to the reliability system. For example, the failure of a component does not change the state of the environment, but, if the external demand is high and the system is loaded, failure probabilities of the components can also be higher due to the dependence on the environment.

Once the failure structure of the network is identified, we can define the transition probability matrix $Q$ accordingly, and this structure may indeed depend on the environment. The form of (22) is only one possibility, and our results in the following sections are still applicable if it has other forms. For example, it may well be the case that in environment $i=1$ one of the two 
parallel components, say 2 , is disengaged. The system then becomes a serial connection of 1 and 3 in environment 1 , and it now suffices to take

$$
Q_{1}(S, M)= \begin{cases}\pi_{1}(1) \pi_{3}(1), & \text { if } M=\{1,2,3\}, \\ \pi_{1}(1)\left(1-\pi_{3}(1)\right), & \text { if } M=\{1,2\}, \\ \left(1-\pi_{1}(1)\right) \pi_{3}(1), & \text { if } M=\{2,3\}, \\ \left(1-\pi_{1}(1)\right)\left(1-\pi_{3}(1)\right), & \text { if } M=\{2\}\end{cases}
$$

since component 2 is not in operation and it cannot fail. The failure structure prescribed through $Q$ is indeed quite general, making it possible to analyze a wide range of reliability models.

\section{NETWORK RELIABILITY ASSESSMENT}

In assessment of network reliability, we are interested in failure free operation of the network for $m$ time periods. More specifically, we want to evaluate $P[L>m]$ for any time $m \geq 0$. Note that we can trivially write

$$
P[L>m]=\sum_{i \in E, S \subseteq \mathscr{K}} P\left[L>m \mid Y_{1}=i, Z_{1}=S\right] P\left[Y_{1}=i, Z_{1}=S\right],
$$

which requires computation of the conditional probability $P\left[L>m \mid Y_{1}=i, Z_{1}=S\right]$ given any initial state $i$ and $S$.

We will denote the conditional network survival probability by

$$
f(i, S, m)=P\left[L>m \mid Y_{1}=i, Z_{1}=S\right],
$$

which is simply the probability that the network will survive $m$ time periods given the set $S$ of initially functioning components and the initial state $i$ of the environment. Similarly, we define the conditional MTTF as

$$
g(i, S)=E\left[L \mid Y_{1}=i, Z_{1}=S\right]=\sum_{m=0}^{+\infty} P\left[L>m \mid Y_{1}=i, Z_{1}=S\right]
$$

We will now exploit the Markov property of the process $(Z, Y)$ to obtain computational results for $f$ and $g$. Once they are computed, it is clear that we obtain the desired results as $f(i$, $\mathscr{K}, m)$ and $g(i, \mathscr{K})$ since it is reasonable to assume that $Z_{1}=\mathscr{K}$ initially.

\subsection{Minimal Repair Model}

In this section, we assume that there is minimal repair and all failed components are replaced only if the whole system fails. The Markov property of $(Z, Y)$ at the first transition yields the recursive formula

$$
f(i, S, m+1)=\sum_{j \in E} \sum_{M \subseteq S, M \in \mathscr{P}} P(i, j) Q_{i}(S, M) f(j, M, m) .
$$


This follows by noting that for the system to survive $m+1$ periods given $\left(Y_{1}=i, Z_{1}=S\right)$, it must first survive the next period by moving to some state $\left(Y_{2}=j, Z_{2}=M(M \subseteq S\right.$ with $\Psi(M)=1$ or $M \in \mathscr{P})$ ) with probability $P(i, j) Q_{i}(S, M)$. Then, being in state $(j, M)$, it must survive the remaining $m$ periods with probability $f(j, M, m)$. The recursive system (28) can be solved for any $(i, S)$ starting with $m=1$ and the boundary condition $f(j, M, 0)=1$ whenever $M \in \mathscr{P}$. Note that we only need to compute $f(i, S, m+1)$ for $S \in \mathscr{P}$ since $f(j, M, m)=$ 0 whenever $M \notin \mathscr{P}$.

Similarly, using (27) or the Markov property directly we obtain the system of linear equations

$$
g(i, S)=1+\sum_{j \in E} \sum_{M \subseteq S, M \in \mathscr{P}} P(i, j) Q_{i}(S, M) g(j, M)
$$

which can be solved easily since both $E$ and $\mathscr{K}$ are finite. Once again, the dimension of the system of linear equations in (29) is reduced by noting that we only need to compute $g(i, S)$ for $S \in \mathscr{P}$ since $g(j, M)=0$ whenever $M \notin \mathscr{P}$.

\subsection{Maximal Repair Model}

We now assume that there is maximal repair and all failed components are replaced at the beginning of each period. This implies that all components are functioning at the beginning of a period and we can take $Z_{1}=S=\mathscr{K}$. Then, (28) can be written as

$$
f(i, \mathscr{K}, m+1)=\sum_{j \in E} \sum_{M \subseteq \mathscr{K}, M \in \mathscr{P}} P(i, j) Q_{i}(\mathscr{K}, M) f(j, \mathscr{K}, m)
$$

with the same boundary condition $f(i, \mathscr{K}, 0)=1$. Note that (30) is dimensionally simpler than (28) since it can be rewritten as

$$
\begin{aligned}
f(i, m+1) & =\left[\sum_{M \in \mathscr{P}} Q_{i}(\mathcal{K}, M)\right] \sum_{j \in E} P(i, j) f(j, m) \\
& =q(i) \sum_{j \in E} P(i, j) f(j, m)
\end{aligned}
$$

after suppressing $\mathscr{K}$ in $f$. Defining the matrix $R(i, j)=q(i) P(i, j)$, we obtain the explicit solution

$$
f(i, m)=\sum_{j \in E} R^{m}(i, j),
$$

where $R^{0}=I$ is the identity matrix. This follows by noting that we can write (32) as $f(\cdot, m+$ 1) $=R f(\cdot, m)$ which implies $f(\cdot, m)=R^{m} f(\cdot, 0)$ through recursion.

A similar analysis on the MTTF yields the system of linear equations

$$
g(i)=1+q(i) \sum_{j \in E} P(i, j) g(j)
$$


which can be written in compact form as $g=1+R g$ with the explicit solution

$$
g(i)=\sum_{j \in E}(I-R)^{-1}(i, j)
$$

For the series system in (10),

$$
q(i)=\sum_{M \in \mathscr{P}} Q_{i}(\mathscr{K}, M)=\prod_{k \in \mathscr{K}} \pi_{k}(i)
$$

and

$$
q(i)=\sum_{M \in \mathscr{P}} Q_{i}(\mathscr{K}, M)=1-\prod_{k \in \mathscr{K}}\left(1-\pi_{k}(i)\right)
$$

for the parallel system in (11).

\section{PRIOR ANALYSIS OF NETWORK RELIABILITY}

The computational formulas provided in the previous section can be used to determine network reliability and the MTTF if the model parameters are known. In what follows, we consider the case where these parameters are unknown and present a Bayesian analysis by describing our uncertainty about the unknown parameters probabilistically. This requires description of our uncertainty about the elements of the parameter vector $\Theta=(\mathbf{P}, \boldsymbol{\pi}(i), i \in E)$. For the components of the transition matrix $\mathbf{P}$ of the environmental process, we assume that the $i$ th row $\mathbf{P}_{i}=\{P(i, j) ; j \in E\}$ has a Dirichlet prior

$$
p\left(\mathbf{P}_{i}\right) \propto \prod_{j \in E} P(i, j)^{\alpha_{j}^{i}-1}
$$

and that the $\mathbf{P}_{i}$ 's are independent of each other. For a given environment $i \in E$, we assume that the elements of the vector $\pi(i)=\left(\pi_{1}(i), \ldots, \pi_{K}(i)\right)$ have independent beta densities

$$
p\left(\pi_{k}(i)\right) \propto\left[\pi_{k}(i)\right]^{a_{k}(i)-1}\left[1-\pi_{k}(i)\right]^{b_{k}(i)-1}
$$

denoted as $\pi_{k}(i) \sim \operatorname{Beta}\left(a_{k}(i), b_{k}(i)\right)$. Also, we assume that $\boldsymbol{\pi}(i)$ 's are independent of each other for all $i \in E$, and they are independent of the components of $\mathbf{P}$. We will denote the prior distribution of $\Theta$ as $p(\Theta)$.

As we will see in the following section, the above specification of the prior distributions provides us with a tractable posterior analysis. These prior distributions yield a conjugate Bayesian analysis, that is, they result in posterior distributions with the same form as the prior but with revised parameters (see Gelman et al. [7] for conjugate Bayesian analysis). When prior information exists about the network failure process and about the behavior of the environmental transitions these can be incorporated into the analysis via the selection of prior parameters. In specifying the prior distributions of $\mathbf{P}_{i}$ 's the prior parameters $\alpha_{j}^{i}$,s can be chosen by specifying a best guess about the transition probability $P(i, j)$ and an uncertainty measure about the best guess from the reliability analysts. The best guess can treated as the mean of $P(i, j)$, which is 
given by $E[P(i, j)]=\alpha_{j}^{i} / \sum_{j \in E} \alpha_{j}^{i}$ for the Dirichlet prior, and the elicited uncertainty measure can be used to obtain the prior variance estimate under the Dirichlet distribution. The mean and variance identities can be solved to obtain the prior parameters $\alpha_{j}^{i}$, s. Similarly, for specifying the prior distributions of $\pi_{k}(i)$ 's, we can use the mean and variance identities for the beta distribution to obtain $a_{k}(i)$ 's and $b_{k}(i)$ 's. Other methods for prior elicitation in beta and Dirichlet distributions are given in Chaloner and Duncan [3].

We note that the network reliability function $f$ in (28) and (32) is a function of $\Theta$, that is, the probability statement about the failure free performance of the network is conditional on the parameter vector $\Theta$. To make this explicit, we will write

$$
f(i, S, m \mid \Theta)=P\left[L>m \mid Y_{1}=i, Z_{1}=S, \Theta\right]
$$

Similarly, the MTTF function $g$ in (29) and (34) will be written as

$$
g(i, S \mid \Theta)=E\left[L>\mid Y_{1}=i, Z_{1}=S, \Theta\right]
$$

We will demonstrate how the prior analysis can be done on network reliability $f$ for the minimal repair model only. The analysis for the case of maximal repair follows along the same lines. Conditional on $\Theta$, we can evaluate the network reliability $f$ under the minimal repair scenario using the recursive formula (28)

$$
f(i, S, m+1 \mid \Theta)=\sum_{j \in E} \sum_{M \subseteq S, M \in \mathscr{P}} P(i, j) Q_{i}(S, M \mid \boldsymbol{\pi}(i)) f(j, M, m \mid \Theta)
$$

with the boundary condition $f(i, S, 0 \mid \Theta)=1$. Then, prior to observing any data, we can make network reliability predictions as

$$
f(i, S, m)=\int f(i, S, m \mid \Theta) p(\Theta) d \Theta .
$$

The above integral cannot be evaluated analytically, but it can be approximated via simulation as a Monte Carlo integral

$$
f(i, S, m) \approx \frac{1}{G} \sum_{g} f\left(i, S, m \mid \Theta^{(g)}\right)
$$

by generating $G$ realizations from the prior distribution $p(\Theta)$. It is clear that similar analysis can be developed for the maximal repair case and also for approximating the MTTF $g$ under both scenarios.

\section{POSTERIOR ANALYSIS OF NETWORK RELIABILITY}

If the system is observed for $n$ time periods, then we can write the likelihood function of the unknown parameters $\Theta=(\mathbf{P}, \boldsymbol{\pi}(i), i \in E)$. The observed data consist of $\mathbf{X}^{n}=\left\{\mathbf{X}_{t} ; t=\right.$ $1, \ldots, n\}$, where $\mathbf{X}_{t}=\left(X_{t}(1), X_{t}(2), \ldots, X_{t}(K)\right)$ and may include $Y^{n}=\left\{Y_{t} ; t=1, \ldots\right.$, 
$n\}$ if the environmental process is observable. Recall that the failure data $\mathbf{X}$ also provides the values $Z^{n}=\left\{Z_{t} ; t=1, \ldots, n+1\right\}$ since $Z_{t+1}=\left\{k=1,2, \ldots, K ; X_{t}(k)=1\right\}$.

Once the network is observed for $n$ periods, our uncertainty about $\Theta$ is revised as described by the posterior distribution $p(\Theta \mid D)$, where $D$ denotes the observed data. In developing the posterior analysis under both minimal and maximal repair scenarios, we will consider two cases based on what the observed data $D$ consist of. Specifically, in the first case we will assume that the environmental process $Y$ will be observed. This may be appropriate for situations where the environment such as weather conditions will be observable and components such as temperature, wind velocity, etc. can be measured during each period. In this case, the observed data will consist of both the component failure history $\mathbf{X}$ and the history of the $Y$ process over $n$ periods. In the second case, we consider the situation where the environment may not be observable due to its complexity, and thus the history of the $Y$ process will not be available for the posterior analysis. In this case the Bayesian analysis of the network reliability presents a structure similar to the hidden Markov models, which were considered by a host of authors such as Robert, Celeux, and Diebolt [14] for analyzing mixture models.

In the minimal repair model, we assume that the system will be observed until its failure without replacing any of its components. In other words, the failed components will not be replaced as long as the system operates and the system will be observed either $n$ periods or until its failure, whichever occurs first. Upon the failure of the system, all failed components will be replaced with new ones. Under this scenario, if $X_{t}(k)=0$, then $X_{\tau}(k)=0$ for all $\tau \geq t$. For the case where the environmental process $Y$ is observable, we can write the likelihood function of $\Theta$ in the minimal repair model as

$$
\mathscr{L}(\Theta ; \mathbf{X}, Y) \propto \prod_{t=1}^{n} P\left(Y_{t-1}, Y_{t}\right)\left\{\prod_{k \in Z_{t+1}} \pi_{k}\left(Y_{t}\right) \prod_{k \in\left(Z_{t} \cap Z_{t+1}^{c}\right)}\left[1-\pi_{k}\left(Y_{t}\right)\right]\right\} .
$$

For the case where $Y$ is not observable, the joint likelihood function of $\Theta$ and $Y^{n}$ is obtained from (45) by excluding the term $P\left(Y_{t-1}, Y_{t}\right)$. Under the minimal repair scenario, we have

$$
Z_{1} \supseteq \cdots \supseteq Z_{n+1}
$$

with $Z_{1}=\mathscr{K}$.

In the maximal repair model, whenever a component fails it will be replaced with a functioning component of the same type. In this scenario, when the process $Y$ is observable the likelihood function of $\Theta$ is given by

$$
\mathscr{L}(\Theta ; \mathbf{X}, Y) \propto \prod_{t=1}^{n} P\left(Y_{t-1}, Y_{t}\right)\left\{\prod_{k \in \mathscr{K}}\left[\pi_{k}\left(Y_{t}\right)\right]^{X_{t}(k)}\left[1-\pi_{k}\left(Y_{t}\right)\right]^{1-X_{t}(k)}\right\} .
$$

Similar to the minimal repair model when $Y$ process is not observable, the likelihood function is obtained from (47) by excluding the term $P\left(Y_{t-1}, Y_{t}\right)$. Note that in (45) and (47), we set $P\left(Y_{0}\right.$, $\left.Y_{1}\right)=1$ when $t=1$, and we observe only $n-1$ transitions of $Y$.

\subsection{Environmental Process Is Observable}

In this case we assume that both component failure data $\mathbf{X}^{n}=\left\{\mathbf{X}_{t} ; t=1,2, \ldots, n\right\}$ and the history of the environmental process $Y$ are observed, that is, $D=\left\{\mathbf{X}^{n}, Y^{n}\right\}=\left\{Z^{n}, Y^{n}\right\}$. 
Thus, the likelihood function of $\Theta$ is given by (45) for the minimal repair scenario and by (47) for the maximal repair scenario.

As noted by Lee, Judge, and Zellner [8] in Bayesian analysis of Markov chains, the Dirichlet prior for $\mathbf{P}_{i}$ 's results in Dirichlet posteriors. Using the Dirichlet prior (38) with likelihood functions (45) and (47), for both models, we can show that the posterior distribution of $\mathbf{P}_{i}$ 's can be obtained as independent Dirichlets given by

$$
\left(\mathbf{P}_{i} \mid D\right) \sim \text { Dirichlet }\left\{\alpha_{j}^{i}+\sum_{t=1}^{n} 1\left(Y_{t}=i, Y_{t+1}=j\right) ; j \in E\right\}
$$

where $1(\cdot)$ is the indicator function. Similarly, since the beta distribution is a conjugate prior for Bernoulli likelihood functions (see, e.g., Gelman et al. [7]), the posterior analysis for component survival probabilities is tractable. Using the beta prior (39) with likelihoods (45) and (47) separately, the posterior distributions of $\pi_{k}(i)$ 's are obtained as independent beta densities given by

$$
\left(\pi_{k}(i) \mid D\right) \sim \operatorname{Beta}\left[a_{k}^{*}(i), b_{k}^{*}(i)\right]
$$

with

$$
\begin{gathered}
a_{k}^{*}(i)=a_{k}(i)+\sum_{t=1}^{n} 1\left(Y_{t}=i\right) 1\left(k \in Z_{t+1}\right), \\
b_{k}^{*}(i)=b_{k}(i)+\sum_{t=1}^{n} 1\left(Y_{t}=i\right) 1\left(k \in\left(Z_{t} \cap Z_{t+1}^{c}\right)\right)
\end{gathered}
$$

for the minimal repair model and with

$$
\begin{gathered}
a_{k}^{*}(i)=a_{k}(i)+\sum_{t=1}^{n} 1\left(Y_{t}=i\right) X_{t}(k), \\
b_{k}^{*}(i)=b_{k}(i)+\sum_{t=1}^{n} 1\left(Y_{t}=i\right)\left(1-X_{t}(k)\right)
\end{gathered}
$$

for the maximal repair model. We note that posteriori elements of $\boldsymbol{\pi}(i)$ 's and $\mathbf{P}$ are independent of each other for all $i \in E$. We also note that the conditional independence of the elements of $\boldsymbol{\pi}(i)$ 's is preserved posterior when the history of the $Y$ process is known. Thus, the posterior distribution $p(\Theta \mid D)$ can be easily evaluated using this independence structure.

Once this posterior analysis is completed we can make reliability predictions conditional on the observed data $D$. In this case the data consists of both the failure data $\mathbf{X}$ and the history of the environmental process $Y$. Thus, we have $p(\Theta \mid D)=p\left(\Theta \mid Z^{n}, Y^{n}\right)$. In obtaining posterior 
network reliability predictions, we want to evaluate $P\left[\hat{L}>m \mid Z^{n}, Y^{n}\right]$, where $\hat{L}=L-n$ is the time remaining to network failure. Note that for the minimal repair case, using the Markov property of the chain $(Y, Z)$, we can write the posterior network reliability as

$$
P\left[\hat{L}>m \mid Z^{n}, Y^{n}\right]=\sum_{j \in E} \int P\left(Y_{n}, j\right) f\left(j, Z_{n+1}, m \mid \Theta\right) p\left(\Theta \mid Z^{n}, Y^{n}\right) d \Theta
$$

where $f\left(j, Z_{n+1}, m \mid \Theta\right)$ is obtained as the solution of (42). The posterior reliability can be approximated as a Monte Carlo integral via

$$
P\left[\hat{L}>m \mid Z^{n}, Y^{n}\right] \approx \frac{1}{G} \sum_{g} \sum_{j \in E} P\left(Y_{n}, j\right) f\left(j, Z_{n+1}, m \mid \Theta^{(g)}\right)
$$

by generating $G$ realizations from the posterior distribution $p\left(\Theta \mid Z^{n}, Y^{n}\right)$. Similarly, we can approximate the posterior prediction for MTTF of the network. For the maximal repair case, we can obtain the results by replacing $Z_{n+1}$ with $\mathscr{K}$ in (42) and all the terms above.

\subsection{Environmental Process Is Unobservable}

When the history of $Y$ process is not observable, we can no longer obtain an analytically tractable posterior analysis as in the previous section. Thus, in what follows we will develop posterior analysis using Markov Chain Monte Carlo methods and, more specifically, using Gibbs sampling (see, e.g., Gelfand and Smith [6]). Assuming the same priors for components of $\Theta$ as in the previous section and defining observed data $D=\left\{\mathbf{X}^{n}\right\}=\left\{Z^{n}\right\}$, a Gibbs sampler can be developed to iteratively draw from the full posterior conditional distributions of all unknown quantities to obtain a sample from, $p\left(\Theta, Y^{n} \mid D\right)$, the joint posterior distribution of $(\Theta$, $\left.Y^{n}\right)$. We note that when the environmental process is unobservable, as pointed out in Section 5, the likelihood functions (45) and (47) are adjusted to obtain the joint likelihood functions of $\Theta$ and $Y^{n}$ for the minimal and maximal repair scenarios.

The implementation of the Gibbs sampler requires the full posterior conditional distributions $\left\{p\left(\mathbf{P}_{i} \mid D, Y^{n}\right) ; i \in E\right\}$ that are independent Dirichlet densities as given by (48), $\left\{p\left(\pi_{k}(i) \mid D\right.\right.$, $\left.\left.Y^{n}\right) ; i \in E, k=1, \ldots, K\right\}$ that are independent beta densities with parameters given by (50)-(53) depending on the repair scenario and $p\left(Y_{t} \mid D, Y^{(-t)}, \boldsymbol{\pi}\left(Y_{t}\right), \mathbf{P}\right)$, where $Y^{(-t)}=\left\{Y_{\tau}\right.$; $\tau \neq t\}$.

For the minimal repair scenario the full posterior conditional distribution of $Y_{t}$ can be obtained by looking at (45) as a function of $Y_{t}$ given everything else. Similar to the development considered by Robert, Celeux, and Diebolt [14], it can be shown that

$$
p\left(Y_{t} \mid D, Y^{(-t)}, \boldsymbol{\pi}\left(Y_{t}\right), \mathbf{P}\right) \propto P\left(Y_{t-1}, Y_{t}\right)\left\{\prod_{k \in Z_{t+1}} \pi_{k}\left(Y_{t}\right) \prod_{k \in Z_{t} \cap Z_{t+1}^{c}}\left[1-\pi_{k}\left(Y_{t}\right)\right]\right\} P\left(Y_{t}, Y_{t+1}\right)
$$

with constant of proportionality

$$
\left[\sum_{j \in E} P\left(Y_{t-1}, j\right)\left\{\prod_{k \in Z_{t+1}} \pi_{k}(j) \prod_{k \in Z_{t} \cap Z_{t+1}^{c}}\left[1-\pi_{k}(j)\right]\right\} P\left(j, Y_{t+1}\right)\right]^{-1}
$$


The full posterior conditional distribution of $Y_{t}$ for the maximal repair case can be obtained by looking at (47) as a function of $Y_{t}$. In this case it can be shown that

$$
p\left(Y_{t} \mid D, Y^{(-t)}, \boldsymbol{\pi}\left(Y_{t}\right), \mathbf{P}\right) \propto P\left(Y_{t-1}, Y_{t}\right)\left\{\prod_{k \in \mathscr{K}}\left[\pi_{k}\left(Y_{t}\right)\right]^{X_{t}(k)}\left[1-\pi_{k}\left(Y_{t}\right)\right]^{1-X_{t}(k)}\right\} P\left(Y_{t}, Y_{t+1}\right)
$$

with constant of proportionality

$$
\left[\sum_{j \in E} P\left(Y_{t-1}, j\right)\left\{\prod_{k \in \mathscr{K}}\left[\pi_{k}(j)\right]^{X_{t}(k)}\left[1-\pi_{k}(j)\right]^{1-X_{t}(k)}\right\} P\left(j, Y_{t+1}\right)\right]^{-1}
$$

For the boundary cases of $t=1$ and $t=n$, one should set $P\left(Y_{0}, Y_{1}\right)=P\left(Y_{n}, Y_{n+1}\right)=1$ in (56)-(59).

For both repair scenarios, a posterior sample from $p\left(\Theta, Y^{n} \mid D\right)$ can be easily obtained by iteratively drawing from the given full posterior conditionals. As we have noted before, the Bayesian analysis we have presented in the above shares common features with the Bayesian analysis of hidden Markov models considered by Robert, Celeux, and Diebolt [14].

The data now consists only of $Z^{n}$ in the above and the posterior distribution is given by $p(\Theta$, $Y^{n} \mid Z^{n}$ ). To obtain posterior network reliability predictions, we want to evaluate $P\left[\hat{L}>m \mid Z^{n}\right]$. Note that for the minimal repair model this can be obtained as

$$
P\left[\hat{L}>m \mid Z^{n}\right]=\sum_{Y^{n}} \int \sum_{j \in E} P\left(Y_{n}, j\right) f\left(j, Z_{n+1}, m \mid \Theta\right) p\left(\Theta, Y^{n} \mid Z^{n}\right) d \Theta
$$

where $f\left(j, Z_{n+1}, m \mid \Theta\right)$ is obtained as the solution of (42). Note that there is no closed form solution for the above integral, but it can be easily approximated by a Monte Carlo integral as

$$
P\left[\hat{L}>m \mid Z^{n}\right] \approx \frac{1}{G} \sum_{g} \sum_{j \in E} P\left(Y_{n}^{(g)}, j\right) f\left(j, Z_{n+1}, m \mid \Theta^{(g)}\right)
$$

by generating $G$ realizations from the posterior distribution $p\left(\Theta, Y_{n} \mid Z\right)$. For the maximal repair scenario we replace $Z_{n+1}$ with $\mathscr{K}$ in (42) and all the terms above. Evaluation of MTTF predictions follows along the same lines.

\section{NUMERICAL ILLUSTRATION}

To illustrate the use of proposed network reliability models and the Bayesian inference procedures for reliability assessment we consider the simple network shown in Figure 1. Assuming that components 1 and 2 are identical and component 3 is different, we considered a scenario which consists of two environments which can be regarded as normal and severe environments, and simulated the probabilistic structure and the component failure/survival process discussed in Section 2. The network was simulated for 365 time periods, and it was assumed that the maximal repair protocol was used. In so doing, the transition matrix 
Components 1 \& 2 in Environment 1

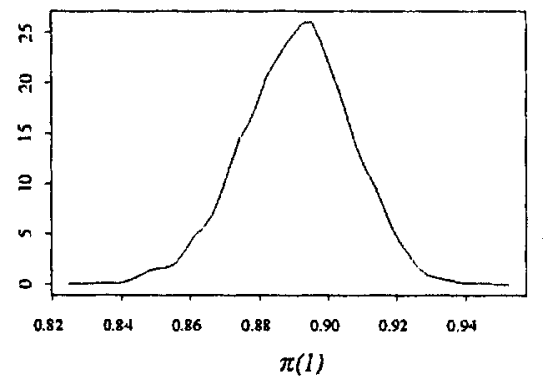

Component 3 in Environment 1

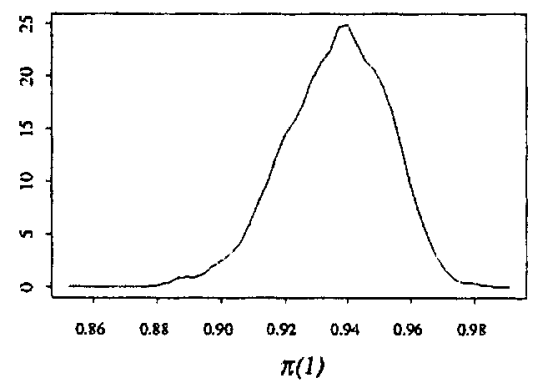

Components 1 \& 2 in Environment 2

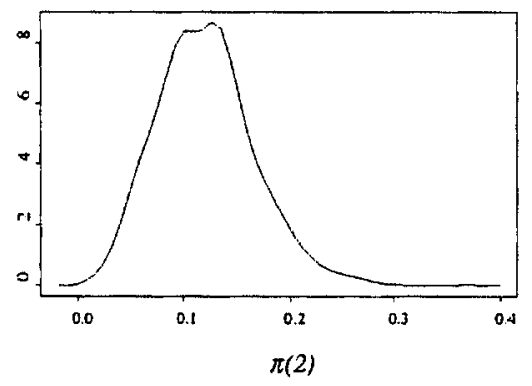

Component 3 in Environment 2

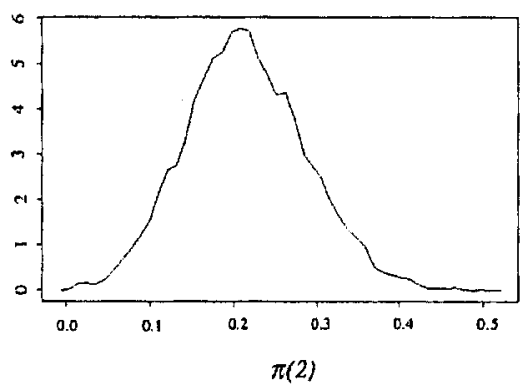

Figure 2. Posterior distributions of component survival probabilities in Environments 1 and 2.

$$
P=\left[\begin{array}{ll}
0.8 & 0.2 \\
0.9 & 0.1
\end{array}\right]
$$

was used in simulating the environmental process with the initial state given as $Y_{1}=1$. Actual component survival probabilities $\pi_{1}(1)=\pi_{2}(1)=0.9$ and $\pi_{3}(1)=0.95$ for environment 1 , and $\pi_{1}(2)=\pi_{2}(2)=0.2$ and $\pi_{3}(2)=0.25$ for environment 2 were used to simulate the failure data. The simulated component failure data are not given here due to space limitations.

In our illustration we assume that the environmental process is not observed, and we use diffused prior distributions for the elements of $\Theta$. More specifically, for the Dirichlet priors in (38) we assume that $\alpha_{j}^{i}=1$ for all $(i, j)$, implying that $E\left[P_{i}\right]=0.5$ for $i=1,2$. For the beta priors of $\pi_{k}(i)$ 's in (39) we assume that $a_{k}(i)=1$ and $b_{k}(i)=1$ for $i=1,2$ and $k=1,2$, 3 , implying that $E\left[\pi_{k}(i)\right]=0.5$. The above choice of parameters implies uniform prior distributions over $(0,1)$ for each of the parameters and represents a high degree of prior uncertainty about components of $\Theta$. In a situation where prior information exists, the prior

Table 1. Posterior means and standard deviations for transition probabilities.

\begin{tabular}{lcc}
\hline$P(i, j)$ & $E[P(i, j) \mid D]$ & $\sqrt{V[P(i, j) \mid D]}$ \\
\hline$P(1,1)$ & 0.811 & 0.030 \\
$P(1,2)$ & 0.189 & 0.030 \\
$P(2,1)$ & 0.926 & 0.043 \\
$P(2,2)$ & 0.074 & 0.043 \\
\hline
\end{tabular}


Table 2. Posterior distributions of selected $Y_{t}$ 's and their actual values.

\begin{tabular}{rccc}
\hline \multicolumn{1}{c}{$t$} & $P\left[Y_{t}=1 \mid D\right]$ & $P\left[Y_{t}=2 \mid D\right]$ & $Y_{t}$ \\
\hline 2 & 0.218 & 0.782 & 2 \\
10 & 0.963 & 0.037 & 1 \\
40 & 0.933 & 0.067 & 1 \\
58 & 0.008 & 0.992 & 2 \\
365 & 0.935 & 0.065 & 1 \\
\hline
\end{tabular}

parameters can be specified by eliciting best guess values for elements of $\Theta$ and uncertainties about these values from reliability analysts. In what follows, we will focus on posterior analysis of the simulated data and discuss how posterior reliability predictions can be obtained.

In Figure 2 we present the density plots for the posterior distributions of component survival probabilities for environments 1 and 2 . We note that, compared to the uniform prior distributions, the posterior distributions are peaked and are concentrated in different regions depending on the environment. In fact, the posterior means of the $\pi_{k}(i)$ 's are very close to the actual values used in simulating the data. For the identical components 1 and 2, the posterior mean of the survival probability is 0.891 and the posterior standard deviation is 0.016 under environment 1 . The posterior mean and standard deviation for component 3 are 0.936 and 0.016 , respectively. The posterior means of survival probabilities are 0.13 for components 1 and 2 and 0.215 for component 3 under environment 2. Note that the posterior distributions under environment 2 have higher variances than the ones under environment 1 . This is expected since the environmental process spends more time in state 1 than in state 2 . We can see this by looking at the posterior distribution of the probability transition matrix $P$ given in Table 1 . We note that the posterior means are very close to the actual values of the transition probabilities given in (62) implying that the environmental process will be in state 1 most of the time.

\section{Reliability Comparison}

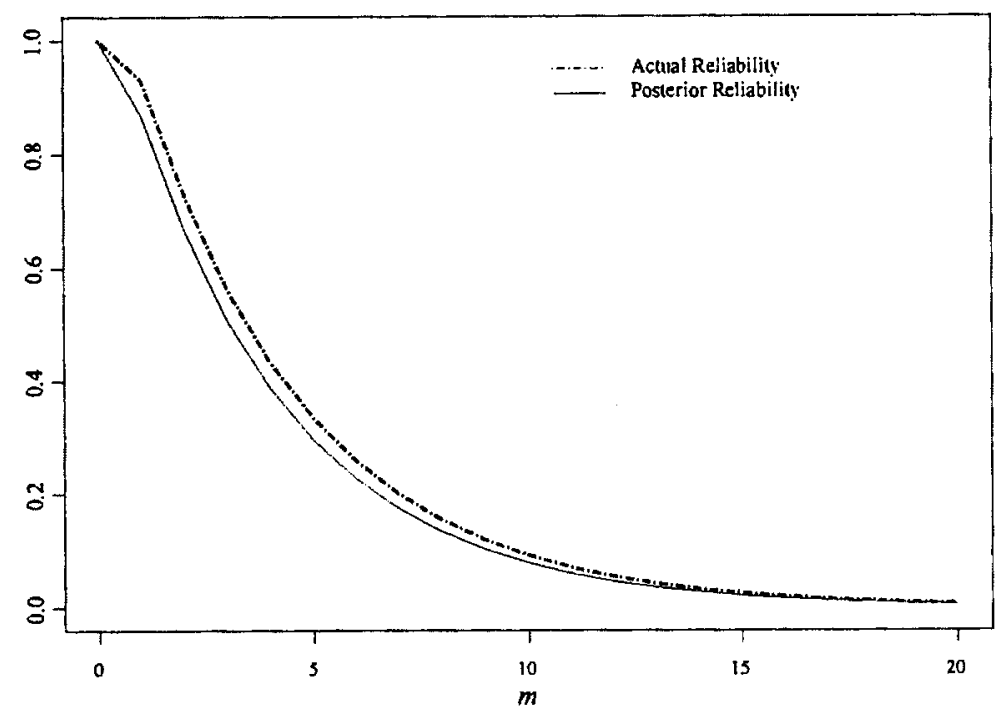

Figure 3. Comparison of actual and posterior reliability functions. 

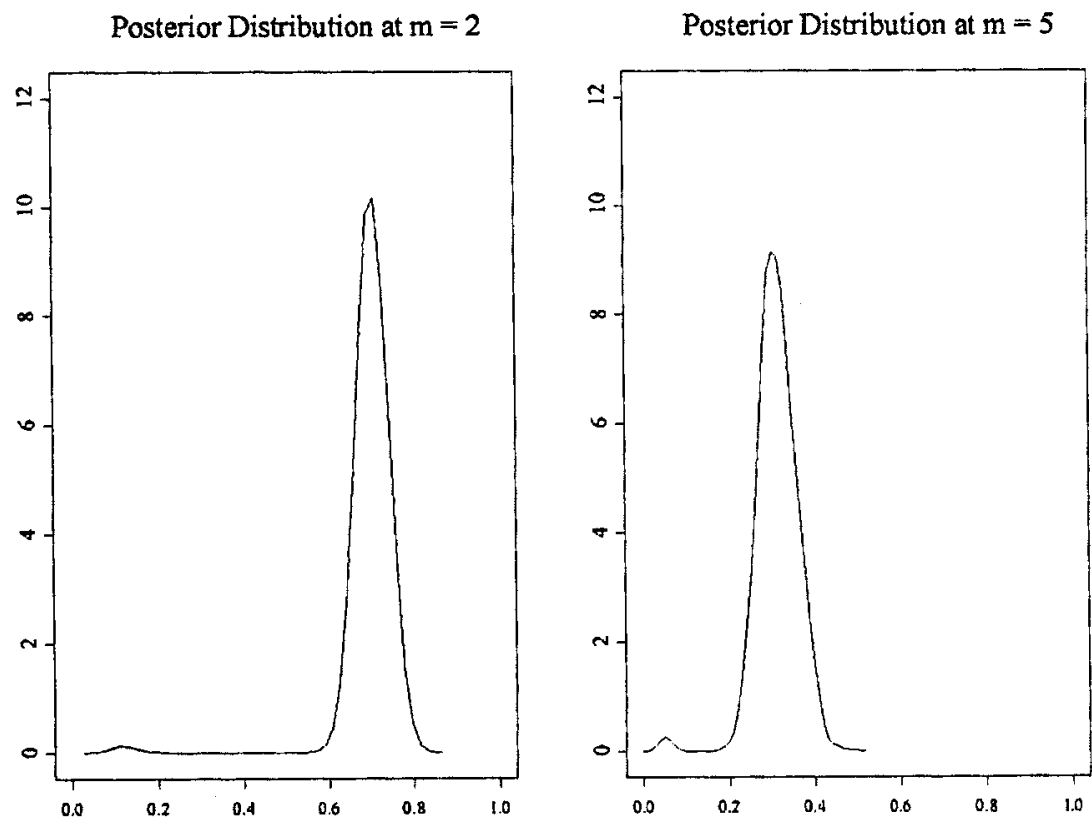

Figure 4. Posterior distributions of reliability for mission times 2 and 5.

The Gibbs sampler enables us to obtain the posterior distributions of the environmental states $Y_{1}, \ldots, Y_{n}$. In Table 2 we present these posterior distributions for selected states as well as their actual (but unobserved) values used in the simulation of the data. For example, at time period 40 the actual value of the unobserved environment is 1 and based on the proposed approach posterior probability that the environmental process is in state 1 is obtained as 0.933 . Table 2 illustrates the posterior probabilities for five periods. The posterior distributions are available for all periods and in almost all cases the posterior probability of the correct state is very high. Thus, the approach is able to infer the unobserved environment correctly for the data.

Once the posterior samples are available, we can make posterior reliability predictions using the Monte Carlo approximation

$$
P\left[\hat{L}>m \mid Z^{n}\right] \approx \frac{1}{G} \sum_{g} \sum_{j \in E} R^{m}\left(Y_{n}^{(g)}, j \mid \Theta^{(g)}\right),
$$

where $R^{m}\left(Y_{n}^{(g)}, j \mid \Theta^{(g)}\right)$ is obtained from (32) for the maximal repair scenario. This requires that $R^{m}\left(Y_{n}, j \mid \Theta\right)$ is evaluated for each realization of $Y_{n}$ and $\Theta$ from the posterior distribution. In Figure 3 we show the posterior reliability function and compare it with the actual reliability function based on the values of $\Theta$ used in simulating the failure process. We note that the posterior reliability function is very close to the actual reliability function. In the Bayesian setup we can also make probability statements about reliability at a given mission time $m$; that is, given data we can look at the posterior distribution of the random variable $\sum_{j \in E} R^{m}\left(Y_{n}, j \mid \Theta\right)$ for fixed $m$ using the posterior samples of $Y_{n}$ and $\Theta$. In Figure 4 we show the posterior distribution of reliability at mission times $m=2$ and 5. At mission time 2, the posterior distribution of reliability is concentrated around 0.7 . As expected the posterior distribution of the 
reliability shifts to the left as mission time increases. We note that the posterior reliability function presented in Figure 3 represents the means of the posterior reliability distributions at different mission times.

\section{ACKNOWLEDGMENT}

This research is supported by Subcontract \#35352-6085 with Cornell University under WO8333-04 from the Electric Power Research Institute and the U.S. Army Research Office.

\section{REFERENCES}

[1] R. Billinton and R.N. Allan, Reliability evaluation of power systems, Plenum Press, New York, 1996.

[2] R. Billinton and K.E. Bollinger, Transmission system reliability using Markov processes, IEEE Trans Power Apparatus Syst 87 (1968), 538-547.

[3] K.M. Chaloner and G.T. Duncan, Assessment of a beta prior distribution: PM elicitation, Statistician 32 (1983), 174-180.

[4] E. Çınlar and S. Özekici, Reliability of complex devices in random environments, Probab Eng Inf Sci 1 (1987), 97-115.

[5] D.P. Gaver, F.E. Montmeat, and A.D. Patton, Power system reliability I-measures of reliability and methods of calculation, IEEE Trans Power Apparatus Syst 83 (1964), 727-737.

[6] A.E. Gelfand and A.F.M. Smith, Sampling-based approaches to calculating marginal densities, J Am Stat Assoc 85 (1990), 398-409.

[7] A. Gelman, J.B. Carlin, H.S. Stern, and D.B. Rubin, Bayesian data analysis, Chapman and Hall, New York, 1995.

[8] T.C. Lee, G.G. Judge, and A. Zellner, Maximum likelihood and Bayesian estimation of transition probabilities, J Am Stat Assoc 63 (1968), 1162-1179.

[9] C. Lefèvre and X. Milhaud, On the association of the lifelengths of components subjected to a stochastic environment, Adv Appl Probab 22 (1990), 961-964.

[10] N. Lynn, N.D. Singpurwalla, and A.F.M. Smith, Bayesian assessment of network reliability, SIAM Rev 40 (1998), 202-227.

[11] S. Özekici, Optimal maintenance policies in random environments, European Journal of Operational Research 82 (1995), 283-294.

[12] S. Özekici, "Complex systems in random environments," Reliability and maintenance of complex systems, S. Özekici (Editor), NATO ASI Series Volume F154, Springer-Verlag, Berlin, 1996, pp. 137-157.

[13] S. Özekici, Markov modulated Bernoulli process, Math Methods Oper Res 45 (1997), 311-324.

[14] C.P. Robert, G. Celeux, and J. Diebolt, Bayesian estimation of hidden Markov chains: A stochastic implementation, Stat Probab Lett 16 (1993), 77-83.

[15] N.D. Singpurwalla, Survival in dynamic environments, Stat Sci 10 (1995), 86-103.

[16] N.D. Singpurwalla and M.A. Youngren, Multivariate distributions induced by dynamic environments, Scand J Stat 20 (1993), 251-261. 